\title{
Familial steroid-sensitive nephrotic syndrome in Southern Israel: clinical and genetic observations
}

\author{
Daniel Landau • Tal Oved • Dan Geiger • Luba Abizov • \\ Hanna Shalev • Ruti Parvari
}

Published online: 20 February 2007

(C) IPNA 2007

Unfortunately the column headings were incorrectly titled. The correct Table 1 is printed below:

Table 1 Patients' characteristics ( $S S$ simple steroid-sensitive, FR frequentrelapsing, $S D$ steroid-dependent, $N A$ not available, $N S$ not significant)

\begin{tabular}{lllll}
\hline \multirow{2}{*}{ Characteristics } & \multirow{2}{*}{ Jews } & \multicolumn{2}{l}{ Bedouins } & \multirow{2}{*}{$P$ value $^{\text {a }}$} \\
\cline { 3 - 4 } & & \multicolumn{3}{c}{ Index family } \\
\cline { 3 - 4 } & 48 & 48 & 11 & \\
Number & $37(78)$ & $38(77)$ & $8(73)$ & NS \\
Male (\%) & $2(4.2)$ & $13(28)$ & NA & $<0.005$ \\
Family history (\%) & $4.8 \pm 3.4$ & $4.9 \pm 2.5$ & $4.4 \pm 2.4$ & NS \\
Age at onset (years) & & & & \\
NS Type & $19(40)$ & $34(71)$ & $4(36)$ & $<0.005$ \\
SS (\%) & $16(33)$ & $10(20)$ & $1(9)$ & 0.08 \\
FR (\%) & $12(25)$ & $4(8)$ & $4(27)$ & 0.13 \\
SD (\%) & $1(2)$ & 0 & $2(18)$ & NS \\
NA (\%) & $17(35)$ & $10(21)$ & $1(10)$ & 0.05 \\
Steroid-sparing therapy (\%) & $8(18)$ & $8(17)$ & $4(36)$ & NS \\
Major complications (\%) & $8(26)$ &
\end{tabular}

${ }^{\text {a }}$ Values refer to comparison between Bs and Js

The online version of the original article can be found at http://dx.doi. org/10.1007/s00467-006-0409-7.

D. Landau $\cdot$ H. Shalev

Department of Pediatrics, Faculty of Health Sciences,

Ben Gurion University of the Negev,

Beer-Sheva, Israel

T. Oved $\cdot$ L. Abizov $\cdot$ R. Parvari

Department of Developmental Genetics and Virology,

Faculty of Health Sciences, Ben Gurion University of the Negev,

Beer-Sheva, Israel

D. Geiger

Computer Science Department, Technion,

Haifa, Israel

D. Landau $(\varangle) \cdot$ H. Shalev

Department of Pediatrics, Soroka Medical Center,

P.O. Box 151, Beer-Sheva 84101, Israel

e-mail: 1daniel@bgu.ac.il 